\title{
Comparative Measurement of Biophysical Parameters in Consideration of Skin Graft Donor Site for Nasal Defects
}

\author{
Joo-Hak Kim, Chang Hwan Ahn, Sungmin Kim, Young Lee ${ }^{1}$, Sang-Ha Oh \\ Departments of Plastic and Reconstructive Surgery and ${ }^{1}$ Dermatology, College of Medicine, Chungnam National University, Daejeon, Korea
}

Background: In planning a skin graft, the texture, color, and size of the recipient and donor site tissues should be considered. Objective: We determined the optimal donor sites for nasal full-thickness skin grafting based on biophysical parameters. Methods: Thirty women over the age of 60 were selected for this study. Four recipient sites (nasal root, dorsum, tip, ala) and three donor sites (preauricle, postauricle, forehead) were considered. Biophysical parameters such as transepidermal water loss (TEWL), capacitance, sebum output, erythema/melanin value, and skin replica technique were tested. Results: The nasal root was correlated with the forehead in terms of TEWL and sebum output. The nasal dorsum was correlated with the preauricle in terms of TEWL, erythema/melanin value, and skin replica measurements. The nasal tip was correlated with the preauricle in terms of TEWL, sebum output, erythema/melanin value, and skin replica measurements. The ala was correlated with the forehead in terms of TEWL and skin replica measurements. Conclusion: The preauricule is the optimal donor site for resurfacing of the nasal dorsum and tip. The forehead is a good

Received March 24, 2017, Revised January 22, 2018, Accepted for publication February 22, 2018

Corresponding author: Sang-Ha Oh, Department of Plastic and Reconstructive Surgery, Chungnam National University Hospital, 282 Munhwa-ro, Jung-gu, Daejeon 35015, Korea. Tel: 82-42-280-7387, Fax: 82-42-280-7384, E-mail: djplastic@cnu.ac.kr

ORCID: https://orcid.org/0000-0003-3734-5005

Young Lee, Department of Dermatology, Chungnam National University Hospital, 282 Munhwa-ro, Jung-gu, Daejeon 35015, Korea. Tel: 82-42-280-7706, Fax: 82-42-280-7706, E-mail: resina20@cnu.ac.kr ORCID: https://orcid.org/0000-0001-9205-1785

This is an Open Access article distributed under the terms of the Creative Commons Attribution Non-Commercial License (http://creativecommons. org/licenses/by-nc/4.0) which permits unrestricted non-commercial use, distribution, and reproduction in any medium, provided the original work is properly cited.

Copyright (c) The Korean Dermatological Association and The Korean Society for Investigative Dermatology donor site for alar defects. For resurfacing of the nasal root, the forehead and postauricle are good choices. (Ann Dermatol 31(1) $1 \sim 5,2019$ )

-Keywords-

Face/physiology, Nose, Skin transplantation

\section{INTRODUCTION}

Nasal defects are commonly caused by trauma or skin cancer. Given that the nose is located in the center of the face and projects from its surface, even a small deformation can be noticeable. For these reasons, when choosing a method for nasal reconstruction both functional and aesthetic factors should be taken into account, including the size and depth of the defect. A variety of methods may be used for nasal reconstruction, including secondary healing, skin grafts, and skin flaps (e.g., local, regional, distant, and free flaps). Skin grafting, one of the most commonly used methods for nasal reconstruction, is highly recommended for superficial defects that do not involve bones or cartilage. Also, it is simple and better reconstruction procedure according to anatomic site, but it can lead to poor aesthetic results due to mismatch of thickness, texture and scar contraction ${ }^{1}$. Therefore, the donor site for such procedures should be selected keeping in mind similarities in color and texture with the recipient site. In the case of nasal skin grafts, the postauricle area is commonly used as a donor site to minimize donor site morbidity (e.g., scarring). However, the nose can be divided into a number of aesthetic subunits with different characteristics, which should be considered when selecting a donor site for nasal reconstruction.

The donor site for nasal resurfacing with a skin graft should be selected based on the actual quality of the skin. 
Biophysical parameters are used to estimate regional variations in facial skin ${ }^{2}$. However, no study of skin grafting has compared nasal skin with donor skin based on biophysical parameters. Therefore, in this study we determined the optimal donor sites for full-thickness skin grafting according to the parts of the nose on the basis of four biophysical parameters: moistness, amount of skin oil, degree of erythema/pigmentation, and wrinkles.

\section{MATERIALS AND METHODS}

A prospective study was conducted among 30 patients who treated in a dermatology from March 2014 to June 2014. This study protocol received approval from the Institutional Review Board of the Chungnam National University Hospital, Daejon, South Korea (IRB no. CNUH 2013-12-011). Thirty women over the age of 60 (a

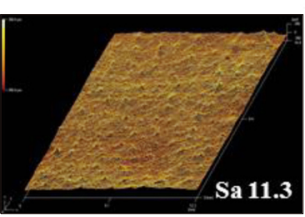

Forehead

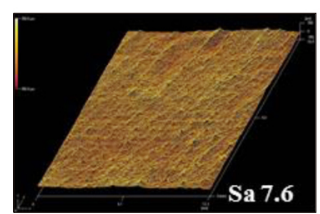

Preauricle region

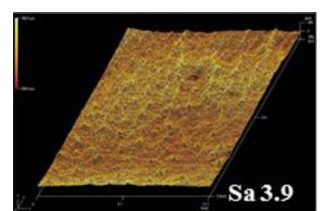

Postauricle region

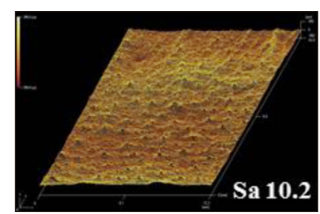

Nasal root

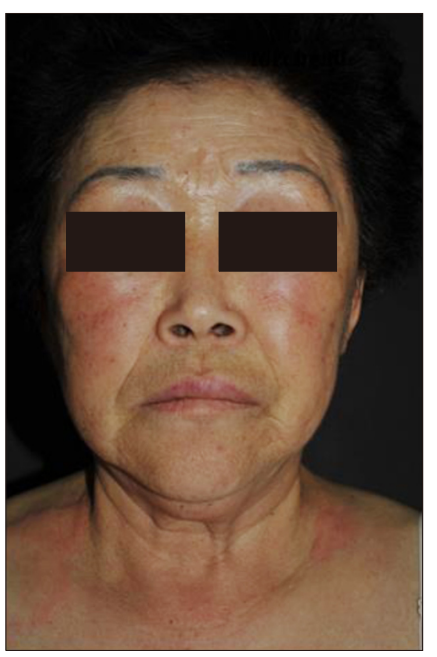

high-risk group for skin cancer) were selected for this study. Written informed consent was obtained from all participants. We received the patient's consent form about publishing all photographic materials. The age profile of the subjects was from 60 to 84 years (mean age, 69.6 years). Four nasal sites (nasal root, dorsum, tip, and ala) and three donor skin sites (preauricle region, postauricle region, and forehead) were considered. Transepidermal water loss (TEWL) and skin hydration (capacitance) were measured to track the moistness of the areas. The amount of skin oil in each area was estimated based on the lipid content of the skin surface (sebum output). A narrow-band reflectance spectrophotometer was used to assess the erythema/melanin value. Wrinkling of the skin was assessed using the skin replica technique. In all cases, the measurements were made by the same investigator in a room with a constant temperature $\left(22^{\circ} \mathrm{C} \sim 24^{\circ} \mathrm{C}\right)$ and humidity level

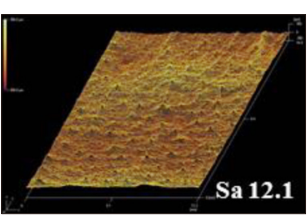

Nasal dorsum

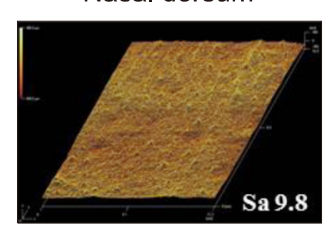

Nasal tip

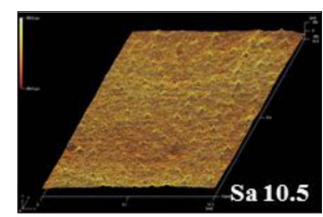

Ala
Fig. 1. A skin replica prepared using a silicone base for wrinkle measurement. Three-dimensional images taken by the Exyma-SK ${ }^{\mathbb{R}}$ (Z-scan). Mean surface roughness (Sa, $\mu \mathrm{m}$ ) was used as a degree of wrinkle indicator.

Table 1. Biophysical parameter values

\begin{tabular}{lrrrrrrr}
\hline & Forehead & \multicolumn{1}{c}{$\begin{array}{c}\text { Preauricle } \\
\text { region }\end{array}$} & $\begin{array}{c}\text { Postauricle } \\
\text { region }\end{array}$ & Nasal root & Dorsum & Nasal tip & Ala \\
\hline TEWL & $10.74 \pm 0.33$ & $11.69 \pm 0.43$ & $9.16 \pm 0.33$ & $11.11 \pm 0.37$ & $11.64 \pm 0.34$ & $11.60 \pm 0.42$ & $11.03 \pm 0.30$ \\
Capacitance & $99.93 \pm 1.19$ & $96.90 \pm 1.13$ & $90.83 \pm 0.91$ & $91.29 \pm 0.95$ & $92.11 \pm 0.86$ & $90.60 \pm 0.93$ & $91.97 \pm 0.97$ \\
Sebum & $74.32 \pm 1.04$ & $71.55 \pm 1.05$ & $66.30 \pm 1.26$ & $75.11 \pm 1.12$ & $75.46 \pm 1.03$ & $77.24 \pm 0.94$ & $77.81 \pm 0.98$ \\
Erythema/pigmentation & $26.64 \pm 0.72$ & $26.29 \pm 0.79$ & $17.79 \pm 0.61$ & $24.36 \pm 0.88$ & $24.07 \pm 1.09$ & $25.14 \pm 1.14$ & $24.79 \pm 0.89$ \\
Skin replica & $10.98 \pm 0.35$ & $9.01 \pm 0.29$ & $7.66 \pm 0.29$ & $7.65 \pm 0.23$ & $8.34 \pm 0.29$ & $10.63 \pm 0.32$ & $10.72 \pm 0.35$ \\
\hline
\end{tabular}

Values are presented as mean \pm standard error. TEWL: transepidermal water loss. 
$(40 \% \sim 45 \%)$.

\section{Methods}

To standardize the condition of the skin before taking measurements, the test area was cleaned with a mild agent. To eliminate the influence of the cleansing process on the skin, the experiment began 2 to $3 \mathrm{~h}$ after cleaning. To measure TEWL, a Tewameter TM $210^{\circledR}$ measuring device (Courage and Khazaka, Cologne, Germany) was used. The results are expressed as $\mathrm{g} / \mathrm{m}^{2} / \mathrm{h}^{\prime}$. The estimated capacitance, based on the water content of the skin, was determined using a Corneometer CM 825 ${ }^{\circledR}$ (Courage and Khazaka). The values are displayed in arbitrary units ${ }^{4}$.

The output of sebum was estimated using a Sebumeter SM $815^{\circledR}$ (Courage and Khazaka) by putting the cassette's film into contact with each area for $30 \mathrm{~s}$. The measured values are expressed as ' $\mathrm{mg} / \mathrm{cm}^{2,5}$.

A Mexameter MX $18^{\circledR}$ (Courage and Khazaka) was used to determine the erythema value (vascularity) and melanin value (pigmentation) by reflectance; all measurements were made in the same place, under the same light conditions, and in a standing position to reduce measurement error $^{6-8}$.

To examine the curvature of the skin surface, Silflo ${ }^{\circledR}$ pre-mixed resin (Flexico Developments Ltd., Potters Bar, UK) was mixed with a catalyst at a 1:1 ratio. The mixture was thinly spread over the skin to be measured, and a replica was peeled off of the area after about $10 \mathrm{~min}$. Each time a skin replica was obtained, it was analyzed for air bubbles. If air bubbles were present, a second replica was prepared. Each finished skin replica was digitized for a three-dimensional contour analysis using Exyma-SK ${ }^{\circledR}$ (Z-scan, Asan, Korea) (Fig. 1). Arithmetical mean roughness $(\mathrm{Sa}, \mu \mathrm{m})$ was used as a degree of wrinkle indicator ${ }^{9,10}$.

\section{Statistical analysis}

A paired t-test analysis was performed to evaluate the average difference between groups after measurement of the four recipient sites and three donor sites. The recipient and donor sites were compared using IBM SPSS Statistics version 19.0 (IBM Co., Armonk, NY, USA). In general, $p$-values less than 0.05 are taken to indicate significant differences; however, $p$-values greater than 0.05 were considered to indicate statistical significance in this study (the higher the value, the greater the significance in similarity).

\section{RESULTS}

Biophysical parameters such as moistness (TEWL and capacitance), amount of skin oil (sebum output), erythema/melanin value, and wrinkles (skin replica technique)

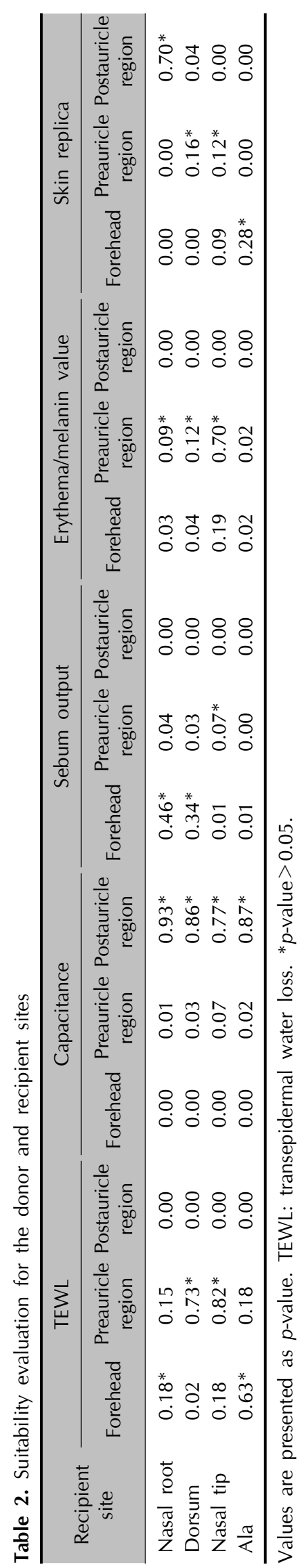


were tested for each donor site on the face and recipient site on the nose (Table 1) and a $p$-value was obtained for each of the test results (Table 2).

According to the $p$-value for each biophysical parameter at the donor sites (forehead, preauricle region, and postauricle region) and recipient sites (nasal root, dorsum, tip, and ala), the highest value from the donor site was selected as the most similar part to each area of the nose. The nasal root was correlated with the forehead in terms of TEWL $(p=0.18)$ and sebum output $(p=0.46)$ and with the postauricle region in terms of the capacitance $(p=$ $0.93)$ and skin replica measurements $(p=0.70)$. The nasal dorsum was correlated with the preauricle region in terms of TEWL $(p=0.73)$, erythema/melanin value $(p=0.12)$, and skin replica measurements $(p=0.16)$. The nasal tip was correlated with the preauricle region in terms of TEWL $(p=0.82)$, sebum output $(p=0.07)$, erythema/melanin value $(p=0.70)$, and skin replica measurements $(p=0.12)$. The ala was correlated with the forehead in terms of TEWL $(p=0.63)$ and skin replica measurements $(p=0.28)$ (Table 2).

The highest value for each biophysical parameter at the donor site was taken to indicate similarity with the recipient site. The forehead and postauricle region were very similar to the nasal root, while the preauricle region was very similar to the nasal dorsum and tip. The alar region was most similar to the forehead.

\section{DISCUSSION}

The nose may be divided into five subunits (dorsum, root, lateral wall, tip, and ala) based on the anatomic outline of the nose, and into three zones based on the thickness of the skin. Zone 1 is soft and thin (the nasal dorsum and lateral wall have nearly no sebaceous glands). Zone 2 is stiff and thick (the nasal tip, supratip area, and ala have numerous sebaceous glands). Like Zone 1, Zone 3 is soft and thin. The alar margin, soft triangle, and columella, which have nearly no sebaceous glands, do not move easily because they are fixed to deeper structures, such as cartilage. In selecting the donor site for a full-thickness skin graft, the preauricular area, which has a thin wall and few sebaceous glands, is suitable for Zones 1 and 3. Given the texture of the skin and the distribution of sebaceous glands, the forehead is a suitable donor site for Zone $2^{11}$. These guidelines are based on anatomical and histological features, but do not reflect the actual state of the skin, such as moistness, amount of skin oil, wrinkles, and erythema. We selected a possible full-thickness skin graft donor site for each part of the nose from the most similar facial area by applying biophysical parameters that reflect the actual state of the skin. These parameters included moistness, indicating the moisture content of the skin surface, amount of skin oil, indicating the functional state of the sebaceous glands, the degree of erythema/pigmentation, reflecting the color of the skin, and wrinkles, indicating the outline of the skin surface.

The stratum corneum maintains skin homeostasis by regulating body fluids and the evaporation of water. The water content of the stratum corneum in a skin graft determines the flexibility of the graft; thus, matching the texture of the skin graft with the microstructure of the skin surface is one of the most important factors in performing a skin graft. TEWL is an indicator of the function of the stratum corneum and reflects the amount of the moisture lost to the outside from the skin, but it does not directly represent the moisture of the stratum corneum and is closely related to the barrier function and moisture preservation function of skin. By determining the rate of diffusion of moisture from the skin surface into the air using Fick's law, the amount of moisture evaporated from the epidermis can be estimated $^{3,12}$. Capacitance can be used as another indicator of moistness. Using the fact that the resistance from the skin surface is inversely related to moisture, one can determine the relative size of the water content by measuring the capacitance $^{4,13}$.

The skin's lipid layer, which is derived from the sebaceous glands or stratum corneum, is an essential feature of skin that affects its barrier function and the evaporation of water and which can be used as a marker of sebum secretion based on the amount of skin oil. The secretion of sebum can be measured based on the fact that if a film gets stained with lipid, the transparency is higher. By measuring the brightness of the film after $30 \mathrm{~s}$ of contact at a constant pressure, the amount of lipid present may be assessed ${ }^{5}$. Vascularity (erythema)/pigmentation can be measured using a spectrophotometer, which takes advantage of the fact that hemoglobin/melanin, which determine skin color, have unique optical absorption spectra. A narrow-band reflectance spectrophotometer is a device for measuring light reflected from the skin surface at wavelengths of 567 $\mathrm{nm}$ (green), $660 \mathrm{~nm}$ (red), and $880 \mathrm{~nm}$ (infrared) were used in this study. By calculating the light intensity absorbed into the skin and reflected from the skin, the erythema and melanin value were calculated. Erythema and melanin value expressed vascularity and pigmentation respectively. And the Mexameter ${ }^{\circledR}$ is an objective skin color-measuring device. It has been reported that this instrument is highly sensitive enough to quantify small differences in skin color $^{6-8}$.

After manufacturing replicas of the skin, quantitative measurements of wrinkles in the skin were made using a du- 
al-wavelength phase meter (an interferometric measuring device for dual-wavelength phase differences). This method of evaluating skin wrinkles has been approved by the Korean Food and Drug Administration ${ }^{9,10}$.

In conclusion, the biophysical parameters at the nasal root, forehead, and postauricle region were very similar. The preauricle region was most similar to the nasal dorsum and nasal tip, while the alar region was most similar to the forehead. These results will be useful in donor site selection for skin grafts in patients with nasal defects.

In this study, biophysical measurements were collected to judge the similarities between full-thickness skin graft recipient sites and donor sites. The results may be used as a meaningful guide for donor site selection. However, the importance of each indicator was not addressed. Also, the evaluations in this study were performed preoperatively using only skin parameters; no evaluations were conducted after placement of the skin graft, postoperatively. Further, this study did not address the effect of sex. Especially, the condition of the skin can be affected by various conditions before surgery and during the healing conditions such as inflammation, ultraviolet (UV) exposure, etc. In that respect, donor and recipient skin cannot be perfect matched by comparative measurement. Additional studies are needed to overcome these limitations.

\section{ACKNOWLEDGMENT}

This work was supported by research fund of Chungnam National University and Basic Science Research Program through the National Research Foundation of Korea (NRF) funded by the Ministry of Science, ICT, \& Future Planning (NRF-2018R1A2B6007425).

\section{CONFLICTS OF INTEREST}

The authors have nothing to disclose.

\section{ORCID}

Joo-Hak Kim, https://orcid.org/0000-0001-9244-0940

Chang Hwan Ahn, https://orcid.org/0000-0003-4182-3823

Sungmin Kim, https://orcid.org/0000-0003-3156-7133

Young Lee, https://orcid.org/0000-0001-9205-1785

Sang-Ha Oh, https://orcid.org/0000-0003-3734-5005

\section{REFERENCES}

1. Chae JK, Kim JH, Kim EJ, Park K. Values of a patient and observer scar assessment scale to evaluate the facial skin graft scar. Ann Dermatol 2016;28:615-623.

2. Marrakchi S, Maibach HI. Biophysical parameters of skin: map of human face, regional, and age-related differences. Contact Dermatitis 2007; 57:28-34.

3. Barel AO, Clarys P. Study of the stratum corneum barrier function by transepidermal water loss measurements: comparison between two commercial instruments: evaporimeter and tewameter. Skin Pharmacol 1995;8:186-195.

4. Blichmann CW, Serup J. Assessment of skin moisture. Measurement of electrical conductance, capacitance and transepidermal water loss. Acta Derm Venereol 1988;68: 284-290.

5. Serup J. Formation of oiliness and sebum output--comparison of a lipid-absorbant and occlusive-tape method with photometry. Clin Exp Dermatol 1991;16:258-263.

6. Yoshimura K, Harii K, Masuda Y, Takahashi M, Aoyama T, Iga T. Usefulness of a narrow-band reflectance spectrophotometer in evaluating effects of depigmenting treatment. Aesthetic Plast Surg 2001;25:129-133.

7. Park ES, Na Jl, Kim SO, Huh CH, Youn SW, Park KC. Application of a pigment measuring device--mexameter-for the differential diagnosis of vitiligo and nevus depigmentosus. Skin Res Technol 2006;12:298-302.

8. Nedelec B, Forget NJ, Hurtubise T, Cimino S, de Muszka F, Legault $A$, et al. Skin characteristics: normative data for elasticity, erythema, melanin, and thickness at 16 different anatomical locations. Skin Res Technol 2016;22:263-275.

9. Ryu JH, Seo YK, Boo YC, Chang MY, Kwak TJ, Koh JS. A quantitative evaluation method of skin texture affected by skin ageing using replica images of the cheek. Int J Cosmet Sci 2014;36:247-252.

10. Hachiya A, Sriwiriyanont $P$, Fujimura $T$, Ohuchi A, Kitahara T, Takema $Y$, et al. Mechanistic effects of long-term ultraviolet $B$ irradiation induce epidermal and dermal changes in human skin xenografts. Am J Pathol 2009;174:401-413.

11. Burget GC. Aesthetic reconstruction of the tip of the nose. Dermatol Surg 1995;21:419-429.

12. Inoue K, Matsumoto K. Quantification of texture match of the skin graft: function and morphology of the stratum corneum. Scand J Plast Reconstr Surg 1986;20:31-35.

13. Hashimoto-Kumasaka K, Takahashi K, Tagami H. Electrical measurement of the water content of the stratum corneum in vivo and in vitro under various conditions: comparison between skin surface hygrometer and corneometer in evaluation of the skin surface hydration state. Acta Derm Venereol 1993;73:335-339. 